\title{
NONIMMERSIONS OF FLAG MANIFOLDS
}

\author{
S. A. ILORI
}

\begin{abstract}
Certain useful quadratic identities in the cohomology of classifying spaces induce quadratic equations in the cohomology of a manifold $M$ under the classifying map for the normal bundle of $M$. In low dimensional flag manifolds, one can show that the quadratic equation has no root, thus establishing a nonimmersion.
\end{abstract}

1. Introduction. In integral cohomology, one has that

$$
p_{r}=\chi^{2} \in H^{4 r}(\mathrm{BSO}(2 r) ; Z)
$$

where $\mathrm{BSO}(2 r)$ is the classifying space for oriented real $r$-plane bundles, $p_{r}$ is the $r$ th Pontryagin class and $\chi$ is the Euler class. An oriented manifold $M$ immerses in codimension $k$ if and only if the stable normal bundle has dimension $k$. (Cf. [3].) We obtain nonimmersion by showing that the quadratic equation in $H^{*}(M ; Z)$, induced by (1.1), has no integral solution.

If $n=n_{1}+\cdots+n_{q}$, let

$$
F\left(n_{1}, \ldots, n_{q}\right)=U(n) / U\left(\bar{n}_{1}\right) \times \cdots \times U\left(n_{q}\right)
$$

denote a complex incomplete flag manifold. We shall show that:

(a) If $M=F(2,4)$, then $\operatorname{dim} M=16, M \subseteq R^{31}, \& R^{24}$.

(b) If $M=F(2,5)$, then $\operatorname{dim} M=20, M \subseteq R^{39}, \& R^{30}$.

The following flag manifolds are bundles with fibre or base equal to $F(2,4)$ or $F(2,5)$ and so results (a) and (b) together with [8] imply the following results.

If $M=F(2,2,2)$, then $\operatorname{dim} M=24, M \subseteq R^{33}, \& R^{32}$.

If $M=F(2,1,3)$, then $\operatorname{dim} M=22, M \subseteq R^{33}, \underline{\&} R^{30}$.

If $M=F(2,2,3)$, then $\operatorname{dim} M=32, M \subseteq R^{46}, \& R^{42}$.

If $M=F(2,3,2)$, then $\operatorname{dim} M=32, M \subseteq R^{46}, \underline{Z} R^{42}$.

If $M=F(2,1,4)$, then $\operatorname{dim} M=28, M \subseteq R^{46}, \subseteq R^{38}$.

If $M=F(1,2,4)$, then $\operatorname{dim} M=28, M \subseteq R^{46}, \& R^{36}$.

If $M=F(1,2,5)$, then $\operatorname{dim} M=34, M \subseteq R^{61}, \underline{Z} R^{44}$.

An interesting aspect of the above results is that the questions of immersion and nonimmersion are now completely solved for the flag manifold $F(2,2,2)$.

The method of this paper was used by Connell [2] to obtain nonimmersions of $F(2,2), F(2,3), F(1,2,2)$ and $F(2,2,2)$. This paper gives an improvement on the result of Connell [2] in the case of the flag manifold $F(2,2,2)$.

It seems that the following conjecture is reasonable.

Received by the editors April 4, 1975 and, in revised form, April 30, 1976.

AMS (MOS) subject classifications (1970). Primary 57A35, 57F15; Secondary 55B15, 55F40.

Key words and phrases. Normal bundle, Chern class, Pontryagin class, Euler class, classifying space.

Copyright $\odot 1977$, American Mathematical Society 
Conjecture. If $M=F(2, n-1)$, then $\operatorname{dim} M=4 n-4, M \subseteq R^{8 n-9}, \underline{Z}$ $R^{6 n-6}$.

2. $F(2,4)$. Let $\mathrm{GL}(n) \rightarrow F(q, n-q)$ be a principal $\mathrm{GL}(q, n-q)$-bundle, $\lambda$, where $\mathrm{GL}(n)$ is the general linear group with entries in the field of complex numbers and $\operatorname{GL}(q, n-q)$ is a subgroup of $\operatorname{GL}(n)$ consisting of all matrices of the form

$$
A=\left[\begin{array}{c|c}
A^{\prime} & B \\
\hline 0 & A^{\prime \prime}
\end{array}\right],
$$

where $A^{\prime} \in \mathrm{GL}(q), A^{\prime \prime} \in \mathrm{GL}(n-q)$ and $B$ is any $q \times(n-q)$-matrix. Let $\lambda^{\prime}, \lambda^{\prime \prime}$ be the subbundle and quotient-bundle associated with $\lambda$. Then $\lambda^{\prime}$ is a canonical $q$-plane bundle over $F(q, n-q)$. By the splitting principle, $\lambda^{\prime}$ splits into line bundles $\xi_{0}, \ldots, \xi_{q-1}$ such that the total Chern class of $\lambda^{\prime}$ is given by

$$
c\left(\lambda^{\prime}\right)=\prod_{i=0}^{q-1} c\left(\xi_{i}\right)=\prod_{i=0}^{q-1}\left(1+\gamma_{i}\right) .
$$

Thus

$$
c_{h}\left(\lambda^{\prime}\right)=\sigma_{h}\left(\gamma_{0}, \ldots, \gamma_{q-1}\right), \quad h=1, \ldots, q,
$$

where $\sigma_{h}=\sigma_{h}\left(\gamma_{0}, \ldots, \gamma_{q-1}\right)$ denotes the $h$ th elementary symmetric function in $\gamma_{0}, \ldots, \gamma_{q-1}$. We also denote by $\bar{\sigma}_{h}=\bar{\sigma}_{h}\left(\gamma_{0}, \ldots, \gamma_{q-1}\right)$ the $h$ th complete symmetric function in $\gamma_{0}, \ldots, \gamma_{q-1}$.

The Whitney sum $\lambda^{\prime} \oplus \lambda^{\prime \prime}$ of the subbundle and quotient-bundle associated with $\lambda$ is the trivial $n$-plane bundle over $F(q, n-q)$. Therefore, $c\left(\lambda^{\prime}\right) c\left(\lambda^{\prime \prime}\right)=$ 1. Thus

$$
c\left(\lambda^{\prime \prime}\right)=\sum_{i=0}^{\infty}(-1)^{i} \bar{\sigma}_{i}\left(\gamma_{0}, \ldots, \gamma_{q-1}\right),
$$

and since $\lambda^{\prime \prime}$ is an $(n-q)$-plane bundle, then

$$
\bar{\sigma}_{i}=\bar{\sigma}_{i}\left(\gamma_{0}, \ldots, \gamma_{q-1}\right)=0, \quad i>n-q .
$$

We also, therefore, have that

$$
\sum_{i=0}^{q} \sigma_{i} \sum_{j=0}^{\infty}(-1)^{j} \bar{\sigma}_{j}=1
$$

from which follow the relations:

$$
\sum_{i=0}^{k}(-1)^{i} \sigma_{i} \bar{\sigma}_{k-i}=0, \quad k>0 .
$$

Now, from $\left[1\right.$, p. 522], we have that the $\sigma_{r}(1 \leqslant r \leqslant q)$ generate $H^{*}(F(q, n-q), Z)$ subject to the relations

$$
\bar{\sigma}_{n-q+i}=0, \quad i \geqslant 0 .
$$

Also, from [9, §1.3] and [5, pp. 354-359], the Chern classes, $c_{i}$, of $F(2,4)$ are given by 


$$
\begin{aligned}
& c_{1}=6 \sigma_{1}, \\
& c_{2}=16 \bar{\sigma}_{2}+18 \sigma_{2}, \\
& c_{3}=26 \bar{\sigma}_{3}+58 \sigma_{2} \sigma_{1}, \\
& c_{4}=31 \bar{\sigma}_{4}+91 \bar{\sigma}_{2} \sigma_{2}+67 \sigma_{2}^{2}, \\
& c_{5}=90 \sigma_{2} \bar{\sigma}_{3}+120 \sigma_{2}^{2} \sigma_{1}, \\
& c_{6}=105 \sigma_{2}^{2} \bar{\sigma}_{2}+65 \sigma_{2}^{3}, \\
& c_{7}=60 \sigma_{2}^{3} \sigma_{1}, \\
& c_{8}=15 \sigma_{2}^{4} .
\end{aligned}
$$

If $F(2,4)$ immerses in codimension 8 , its stable normal bundle, $\nu$, is an $\mathrm{SO}(8)$ bundle. If $d_{i}$ denotes the $i$ th Chern class of $\nu$, then using the identities

$$
\left(\sum_{i=0}^{8} c_{i}\right)\left(\sum_{i=0}^{8} d_{i}\right)=1, \quad \sum_{i=0}^{k} c_{k-i} d_{i}=0, \quad k>0,
$$

and relations (2.1) and (2.2) when $q=2, n=6$, we obtain the following values of $d_{i}, 1 \leqslant i \leqslant 8$.

$$
\begin{aligned}
& d_{1}=-6 \sigma_{1}, \\
& d_{2}=20 \bar{\sigma}_{2}+18 \sigma_{2}, \\
& d_{3}=-50 \bar{\sigma}_{3}-82 \sigma_{2} \sigma_{1}, \\
& d_{4}=105 \bar{\sigma}_{4}+237 \sigma_{2} \bar{\sigma}_{2}+129 \sigma_{2}^{2}, \\
& d_{5}=-546 \sigma_{2} \bar{\sigma}_{3}-504 \sigma_{2}^{2} \sigma_{1}, \\
& d_{6}=1323 \sigma_{2}^{2} \bar{\sigma}_{2}+595 \sigma_{2}^{3}, \\
& d_{7}=-2100 \sigma_{2}^{3} \sigma_{1}, \\
& d_{8}=2100 \sigma_{2}^{4} .
\end{aligned}
$$

Then from [4, Theorem 4.5.1], the Pontryagin class, $p_{4}(\nu)$, of $\nu$ can be computed from the formula

$$
p_{4}(\nu)=2 d_{8}-2 d_{7} d_{1}+2 d_{6} d_{2}-2 d_{5} d_{3}+d_{4}^{2}
$$

to give that $p_{4}(\nu)=-81 \sigma_{2}^{4}$.

Now let $\chi=a \bar{\sigma}_{4}+b \sigma_{2} \bar{\sigma}_{2}+c \sigma_{2}^{2}$ be an element of $H^{8}(F(2,4) ; Z)$; then

$$
\chi^{2}=\left(a^{2}+b^{2}+c^{2}\right) \sigma_{2}^{4} .
$$

Then identity (1.1) implies that $a^{2}+b^{2}+c^{2}=-81$. This quadratic equation has no integral solution. Thus nonimmersion in codimension 8 is established. Therefore, together with [3], we have

Proposition 2.3. The sixteen dimensional manifold $F(2,4)$ does not immerse in codimension 8 but does immerse in codimension 15. 


\section{3. $F(2,5)$.}

Proposition 3.1. The twenty dimensional manifold $F(2,5)$ does not immerse in codimension 10 but does immerse in codimension 19.

Proof. The method of proof is completely the same as for Proposition 2.3 and the details are, therefore, omitted. Note that the Chern classes of $F(2,5)$ are contained in [6, Proposition 3.3] and if $F(2,5)$ immerses in codimension 10 , one finds that the Pontryagin class $p_{5}(\nu)$ of its stable normal bundle $\nu$ is equal to $-504 \sigma_{2}^{5}$.

\section{REFERENCES}

1. A. Borel and F. Hirzebruch, Characteristic classes and homogeneous spaces. I, Amer. J. Math. 80 (1958), 458-538. MR 21 \# 1586.

2. Fred J. Connell, Nonimmersions of low dimensional flag manifolds, Proc. Amer. Math. Soc. 44 (1974), 474-478. MR 49 \# 8037.

3. M. Hirsch, Immersions of manifolds, Trans. Amer. Math. Soc. 93 (1959), 242-276. MR 22 \#9980.

4. F. Hirzebruch, Topological methods in algebraic geometry, 3rd ed., Springer-Verlag, Berlin and New York, 1966. MR 34 \#2573.

5. W. V. D. Hodge and D. Pedoe, Methods of algebraic geometry, Vol. II, Cambridge Univ. Press, New York, 1952. MR 13, 972.

6. S. A. Ilori, Canonical systems on flag manifolds, Atti Accad. Naz. Lincei Rend. Cl. Sci. Fis. Mat. Natur. 17 (1974), 10-17.

7. Séminaire C. Chevalley, 2e année, Anneaux de Chow et applications, Secrétariat math., Paris, 1958. MR 22 \#1572.

8. J. Tornehave, Immersions of complex flag manifolds, Math. Scand. 23 (1968), 22-26 (1969). MR $40 \# 4970$.

9. E. Vesentini, Construction géométrique des classes de Chern de quelques variétés de Grassmann complexes, Colloque de topologie algébrique, Louvain, 1956, 97-120; Thone, Liège; Masson, Paris, 1957. MR 21 \# 1585.

Department of Mathematics, University of Ibadan, Ibadan, Nigeria 